\title{
LAS COLECCIONES DE LEGUMINOSAS EFECTUADAS POR GEORGE B. HINTON, DEPOSITADAS EN EL HERBARIO DE ROYAL BOTANIC GARDENS, KEW
}

\author{
María de Lourdes Rico Arce ${ }^{1}$, Amparo Rodríguez ${ }^{1}$ y Elizabeth Moreno- \\ GuTIÉRREZ ${ }^{2}$
}

${ }^{1}$ Royal Botanic Gardens, Kew, Richmond, Surrey, TW9 3AB, United Kingdom. 1.rico@kew.org, A.rodriguez@kew.org

${ }^{2}$ Comisión Nacional para el Conocimiento y Uso de la Biodiversidad, Avenida Liga Periférico-Insurgentes Sur Núm. 4903, Col. Parques del Pedregal, 1410 Tlalpan, México, D.F. México. emoreno@xolo.conabio.gob.mx

\section{RESUMEN}

Se elaboró una base de datos de los especímenes de la familia Leguminosae colectados por George Boole Hinton en México y depositados en la colección del Herbario de Kew. En este trabajo se proporciona una lista de las especies ordenadas por subfamilia, indicando los números de colecta de Hinton y el material tipo. La base completa está disponible en la Red Mundial de Información Sobre Biodiversidad y en el HerbCat del Herbario de Kew.

Palabras clave: base de datos, colecciones históricas, Hinton, Leguminosae, México.

\begin{abstract}
A database of specimens of the Legume family collected by George Bool Hinton and deposited in the Herbarium at Kew was elaborated. A summary of these collections is given; it includes a check-list of species ordered by subfamily, indicating Hinton's collection numbers and type material. The complete database is available to consult in the Global Net on Biodiversity Information and the HerbCat, Kew.
\end{abstract}

Key words: database, Hinton, historical collections, Leguminosae, Mexico. 


\section{INTRODUCCIÓN}

George B. Hinton fue un naturalista intuitivo y un colector generalista. Nació en Londres en 1882, sus padres fueron Charles Howard Hinton, profesor de matemáticas y novelista, y Mary Everest Boole, también matemática y escritora.

En 1889, a la edad de siete años, con la familia dejó Inglaterra para nunca más volver. Vivió en el Japón y después en los Estados Unidos. En 1911 emigró a México, donde se desempeñó como ingeniero civil, ensayista, ingeniero metalúrgico, arquitecto, industrial y finalmente como colector botánico.

De acuerdo con los archivos de Kew, mientras trabajaba como ingeniero metalúrgico en la mina El Rincón, en Temascaltepec, recibió una propuesta del director de Kew Gardens, Sir Arthur Hill, para recolectar especímenes de plantas.

Sus primeros ejemplares fueron obtenidos por algunos indígenas de la región; principalmente por Luis Martínez y Jerónimo García. Más tarde su hijo menor Jaime le acompañó y efectuó una parte de las exploraciones.

Hinton descubrió seis géneros y 360 especies para la ciencia; y unas 100 de estas últimas se nombraron en su honor. De cada número de colecta acostumbraba preparar de 6 a 12 tantos para distribuir a diversos herbarios. Se estima que la cantidad total de especímenes obtenidos por Hinton, incluyendo duplicados y los que se perdieron por daño de insectos fue de poco menos de 150,000 (Hinton y Rzedowski, 1972). La recolección de ejemplares y su venta era una forma de trabajo remunerado y agradable a la vez, lo cual se refleja en la calidad y preparación de sus especímenes. Vendió sus colecciones principalmente a Inglaterra, Suiza y los Estados Unidos.

Los cuatro años más productivos fueron los transcurridos entre $1936 \mathrm{y}$ 1940. Hinton mismo distribuyó sus especímenes a los herbarios de Kew, Museo Británico, Zurich, Ginebra, Instituto Smithsoniano y la Universidad de Harvard. Eventualmente también envió ejemplares al Field Museum, así como a los jardines botánicos de Missouri y Nueva York. En menor escala repartió sus duplicados a otras instituciones como la Universidad Católica en Washington y Naturhistoriska Rijksmuseum en Holanda. En ocasiones sus muestras fueron adquiridas por colecciones privadas, como la de la Sra. Douglas en la Ciudad de México (Hinton y Rzedowski, 1975).

Una cantidad relativamente pequeña de duplicados se encuentra en el Herbario Nacional del Instituto de Biología, de la Universidad Nacional Autónoma de México y en el Herbario de la Escuela Nacional de Ciencias Biológicas, del Instituto Politécnico Nacional, en México. 
A partir de 1941, durante la Segunda Guerra Mundial, Hinton y su hijo no realizaron trabajo de campo para la recolección de ejemplares botánicos. Por otro lado, en la casa de Hinton las condiciones de almacenamiento no eran controladas, por lo que muchos de los especímenes se perdieron debido al ataque de insectos. Hinton falleció en 1943 como resultado de un accidente de carretera. Después de su muerte, su hijo Jaime envió 56,000 ejemplares, incluyendo duplicados, al Jardín Botánico de Nueva York, los cuales fueron distribuidos posteriormente a diversos herbarios (Hinton y Rzedowski, 1975). En la actualidad, el herbario particular George Hinton cuenta con ca. 8000 ejemplares recolectados por él, por su hijo y por su nieto.

En los archivos de la biblioteca de Kew Gardens se localizan tres carpetas con documentos fechados de 1932 a 1939; éstas contienen cartas, recibos, listas de especímenes y localidades de algunas de sus colecciones, así como también el acuse de recibo por parte de Kew de plantas vivas (orquídeas y helechos) y de materiales secos, frutos (incluyendo bellotas), semillas y maderas.

Hinton siempre procuró cumplir con los requisitos del gobierno de México, depositando un juego de sus colectas en el país; lamentablemente la mayoría de las veces éstas fueron rechazadas tanto por falta de espacio en el Instituto de Biología, como por hostilidad y sospecha por parte de las autoridades del Instituto Biotécnico (Hinton y Rzedowski, 1975).

Hinton trató de utilizar una numeración cronológica en las colecciones, pero durante los últimos cuatro años en que su hijo Jaime colaboró con él y exploró aparte, esto fue difícil de mantener. En 1936, Mr. A. A. Bullock, botánico de Kew, se encargó de la identificación de la mayoría de sus colecciones, particularmente las del género Bursera (Burseraceae); otras muestras fueron identificadas por especialistas de familias diversas también en Kew (Dunk, 1936b).

Sus colecciones tienen un valor científico alto, debido a que la vegetación de las localidades que exploró corresponde en gran parte a bosques tropicales deciduos, donde Hinton estuvo dispuesto a colectar las mismas plantas (árboles o arbustos) en varios estados de desarrollo y en diferentes épocas del año (Dunk, 1935,1936 a y 1936b). Esto es sumamente importante y valioso, por ejemplo para el género Bursera y en general para muchas Anacardiaceae, Styracaceae y representantes leñosos de otras familias.

Hinton concentró su trabajo de campo en los estados de México, Guerrero y Michoacán, cubriendo alrededor de 68,000 kilómetros cuadrados; gran parte de este terreno era botánicamente desconocido debido a lo accidentado del terreno y 
a la inseguridad de los caminos. Muchas de las áreas que exploró, hoy en día son lugares urbanizados, donde la vegetación original ha sido sustituida por asentamientos humanos y aprovechamientos agropecuarios intensivos, de manera que en algunos casos las especies ya no existen en los lugares correspondientes. Hinton y Rzedowski (1972) proporcionaron una lista de las localidades de colecta con las coordenadas geográficas y altitudes respectivas.

En este trabajo se integra una lista de las especies de leguminosas colectadas por George Boole Hinton y sus asociados, depositadas en el Herbario de Kew, ordenadas por subfamilias, indicando los números de colecta y el material tipo.

\section{MÉTODO}

Se revisó la colección completa de leguminosas del Herbario de Kew y los datos de las etiquetas de las plantas de Hinton se incorporaron en una base de datos usando el programa Access 2000. Los autores de las especies fueron estandarizados de acuerdo con Brummitt y Powell (1992). Los nombres de los ejemplares corresponden a la identificación con la cual se localizaron en la mencionada colección. Para denotar el material tipo se apuntó entre paréntesis (tipo), sin definirlo como paratipo, holotipo, isotipo, sintipo.

\section{RESULTADOS}

Las colectas inventariadas fueron realizadas de 1931 a 1941 y algunas en 1969, todas son de México y corresponden a los estados de Guerrero, Michoacán, México, Morelos y Nuevo León. Cabe señalar que aquellas efectuadas en Morelos y Nuevo León, en realidad fueron hechas por su hijo Jaime Hinton. En total se encontraron 1537 ejemplares de herbario, en los que se incluyen 85 géneros, 415 especies y 98 taxones infraespecíficos (ver Apéndice). Las cifras para cada subfamilia se muestran en el Cuadro 1. La lista de especies cuyos tipos corresponden a colectas de Hinton se presenta en el Cuadro 2. Todas las colecciones, incluyendo duplicados, se encuentran ahora disponibles para su consulta en los sitios web de la Red Mundial de Información Sobre Biodiversidad (CONABIO) y el HerbCat del Herbario de Kew. 
Cuadro 1. Colecciones de leguminosas de G. B. Hinton, depositadas en el Herbario de Royal Botanic Gardens, Kew.

\begin{tabular}{lcccc}
\hline Subfamilia & Especímenes & Géneros & Especies & Tipos \\
\hline Caesalpinioideae & 182 & 9 & 53 & 11 \\
Mimosoideae & 359 & 20 & 83 & 5 \\
Papilionoideae & 996 & 56 & 279 & 45 \\
Total: & 1537 & 85 & 415 & 61 \\
\hline
\end{tabular}

Cuadro 2. Lista de las especies de Leguminosae cuyos tipos corresponden a ejemplares de G. B. Hinton, con la cita bibliografía de la descripción original.

Acacia sousae L. Rico, Brittonia 39: 130 (1987). = Acaciella sousae (L. Rico) L. Rico Aeschynomene hintonii Sandwith, Hooker's Icon. Pl. 35: t. 3448 (1943).

Adenopodia gymnantha Brenan, Kew Bull. 41: 87 (1986).

Astragalus hintonii Barneby, Mem. New York Bot. Gard. 13: 156 (1964).

Brongniartia cuneata L.B. Smith \& B.G. Schub., Contr. Gray Herb. 127: 23 (1939).

Brongniartia funiculata L.B. Smith \& B.G.Schub., Contr. Gray Herb. 127: 24 (1939).

Caesalpinia hintonii Sandwith, Kew Bull. 5: 303 (1937).

Canavalia hirsutissima J.D. Sauer, Brittonia 16: 136, f. 11 (1964).

Cassia hintonii Sandwith, Bull. Misc. Inform. Kew 1936 (1): 5 (1936).

Cassia multifoliolata P.G. Wilson, Kew Bull. 13(1): 159 (1958). = Senna multifoliolata

(P.G. Wilson) H.S. Irwin \& Barneby var. multifoliolata

Cassia stenosepala L.B. Sm. \& B.G. Schub., Contr. Gray Herb. 127: 21 (1939). = Senna foetidissima (G. Don) H.S. Irwin \& Barneby var. foetidissima

Cassia tortuosa L.B. Sm. \& B.G. Schub., Contr. Gray Herb. 127: 21 (1939). = Senna pallida (Vahl) H.S. Irwin \& Barneby var. trichocraspedon (Sandwith) H.S. Irwin \& Barneby

Cassia trichocraspedon Sandw., Bull. Misc. Inform. Kew 1936: 2 (1936) ). = Senna pallida

(Vahl) H.S. Irwin \& Barneby var. trichocraspedon (Sandwith) H.S. Irwin \& Barneby

Cloebulia crassistyla R.H. Maxwell, Phytologia 61: 361 (1982).

Coursetia hintonii Rudd, Phytologia 25: 258 (1973).

Crotalaria pilosa Mill. var. robusta H. Senn, Rhodora 41: 331 (1939). = Crotalaria bupleurifolia Cham. \& Schltdl. var. robusta (H. Senn) Windler, Phytologia 21: 265 (1971). 
Cuadro 2. Continuación.

Crotalaria tuerckheimii H. Senn var. macrantha H. Senn, Rhodora 41: 335 (1939).

Dalea cuniculo-caudata P. Wilson, Kew Bull. 13(1): 158 (1958).

Dalea hintonii Sandwith, Bull. Misc. Inform. Kew 1936: 2 (1936).

Erythrina mexicana Krukoff, Brittonia 3: 309 (1939).

Inga hintonii Sandwith, Bull. Misc. Inform. Kew 5: 304 (1937).

Lonchocarpus hintonii Sandwith, Misc. Inform. Kew 1936: 4 (1936).

Lupinus aschenbornii S. Schauer var. cervantesii C.P. Sm., Sp. Lupinorum 4: 58 (1938).

Lupinus decaschistus C.P. Sm., Sp. Lupinorum 16: 248 (1941).

Lupinus elegans Kunth var. quercetorum C.P. Sm., Sp. Lupinorum 8: 115 (1939).

Lupinus fictomagnus C.P. Sm., Sp. Lupinorum 4: 116 (1938).

Lupinus hintonii C.P. Sm., Sp. Lupinorum 4: 63 (1938).

Lupinus monteportae C.P. Sm., Sp. Lupinorum 8: 117 (1939).

Lupinus octablomus C.P. Sm., Sp. Lupinorum 16: 250 (1941).

Lupinus persistens Rose var. praeclarus C.P. Sm., Sp. Lupinorum 4: 59 (1938).

Lupinus quercuum C.P. Sm., Sp. Lupinorum 8: 62 (1938).

Lupinus quercuum C.P. Sm. var. perflexolobius C.P. Sm., Sp. Lupinorum 8: 114 (1939).

Lupinus rhodanthus C.P. Sm., Sp. Lupinorum 8: 115 (1939).

Lupinus simulans Rose var. abietum C.P. Sm., Sp. Lupinorum 4: 64 (1938).

Lupinus squamecaulis C.P. Sm., Sp. Lupinorum 5: 67 (1938).

Lupinus taurimortuus C.P. Sm., Sp. Lupinorum 5: 66 (1938).

Mimosa egregia Sandwith, Bull. Misc. Inform. Kew (1936): 7 (1936).

Nissolia hintonii Sandwith, Hooker's Icon. Pl. 35: t. 3248 (1934).

Nissolia leiogyne Sandwith, Bull. Misc. Inform. Kew (1937): 302 (1937).

Petalostemon tripetalus P.G. Wilson, Kew Bull. 13: 159 (1958). = Dalea urceolata

Greene var. tripetala (P.G. Wilson) Barneby

Phaseolus hintonii A. Delgado, Syst. Bot. 25: 431 (2000).

Piscidia grandifolia (Donn. Sm.) I.M. Johnston var. glabrescens Sandwith, Bull. Misc. Infor. Kew 3 (1936).

Platymiscium jejunum B.B. Klitgaard, Kew Bull. 54: 968, f. 1(1999).

Platymiscium lasiocarpum Sandwith, Hooker's Icon. Pl. 33: t. 3249 (1934).

Senna multifoliolata (P.G. Wilson) H.S. Irwin \& Barneby var. metaxi H.S. Irwin \& Barneby, Mem. New York Bot. Gard. 35: 297 (1982).

Senna pallida (Vahl) H.S. Irwin \& Barneby var. triquetripes H.S. Irwin \& Barneby, Mem. New York Bot. Gard. 35: 552 (1982). 
Cuadro 2. Continuación.

Tephrosia mexicana C.E. Wood, Rhodora 51(611): 344 (1949).

Tephrosia pogonocalyx C.E. Wood, Rhodora 51(611): 341 (1949).

Tephrosia vernicosa C.E. Wood, Rhodora 51(611): 339 (1949).

\section{AGRADECIMIENTOS}

Las autores desean agradecer al Dr. A. Delgado y a la M. en C. Leticia Torres C. por la gentileza que tuvieron en revisar los nombres de los géneros: Desmodium, Phaseolus, Ramirezella y Vigna. Al Dr. Mario Sousa por sus valiosos comentarios y adiciones, a dos revisores y el Prof. J. Rzedowski que con sus atinadas observaciones mejoraron la calidad de este artículo.

\section{LITERATURA CITADA}

Brummitt, R. K. y C. E. Powell. 1992. Authors of plant names. Royal Botanic Gardens, Kew. $732 \mathrm{pp}$.

Dunk, E. G. 1935. Editor notes. Bull. Misc. Inform. Kew. 1935(10): 632.

Dunk, E. G. 1936a. Editor notes. Bull. Misc. Inform. Kew. 1936(1): 1-16.

Dunk, E. G. 1936b. Editor notes. Bull. Misc. Inform. Kew. 1936(6): 346-387.

Dunk, E. G. 1936c. Editor notes. Bull. Misc. Inform. Kew. 1936(6): 387-392

Hinton, J. y J. Rzedowski, J. 1972. George B. Hinton, collector of plants in Southwestern Mexico. J. Arnold Arb. 53: 141-181.

Hinton, J. y J. Rzedowski. 1975. George B. Hinton, explorador botánico en el sudoeste de México. Anales Esc. Nac. Cienc. Biol., México 21: 3-114.

Recibido en abril de 2006.

Aceptado en abril de 2008. 


\section{APÉNDICE}

Lista de las colecciones de Leguminosas de G. B. Hinton, depositadas en el Herbario de Kew.

\section{Subfamilia Caesalpinioideae}

Bauhinia divaricata L. - 12085, 12537, 12559, 16113

Bauhinia latifolia Cav. - 10959, 12289

Bauhinia pauletia Pers. - 1990, 5197, 5691, 6619

Bauhinia pringlei S. Watson - 3579, 5384, 5738

Bauhinia seleriana Harms - 8570

Bauhinia sp. - 10798

Bauhinia subrotundifolia Cav. - 16217

Bauhinia ungulata L. - 2987, 5297, 6945, 11655, 16240

Caesalpinia acapulcensis Standl. - 10991, 16198

Caesalpinia cacalaco Bonpl. - 13366, 16199

Caesalpinia coriaria (Jacq.) Willd. - 1839, 2221, 5851, 6121

Caesalpinia eriostachys Benth. - 5659, 6929, 16216

Caesalpinia hintonii Sandwith - 5567 (tipo), 7746 (tipo)

Caesalpinia pulcherrima Sw. - 733, 945, 4594, 5104, 5875, 6666, 6955, 10049

Caesalpinia vesicaria L. - 10865, 10992

Caesalpinia violacea (Mill.) Standl. - 12064

Cassia grandis L. f. - 5905, 6419, 7156, 7598

Cassia hintonii Sandwith - 3440, 5567 (tipo) 5570, 7727, 7746 (tipo)

Cercis canadensis L. - 17051

Chamaecrista absus (L.) H.S. Irwin \& Barneby var. absus - 2285, 4834, 5053

Chamaecrista desvauxii (Collad.) Killip - 11192

Chamaecrista flexuosa (L.) Greene - 4575, 9070, 10000

Chamaecrista flexuosa (L.) Greene var. texana (Benth.) H.S. Irwin \& Barneby - 6122

Chamaecrista glandulosa Greene var. glandulosa - 10698, 12708

Chamaecrista hispidula (Vahl) H.S. Irwin \& Barneby - 5037, 7029, 9543, 11019, 11591

Chamaecrista kunthiana (Schltdl. \& Cham.) H.S. Irwin \& Barneby - 11388

Chamaecrista nictitans (L.) Moench var. glabrata (Vogel) H.S. Irwin \& Barneby - 10841

Chamaecrista nictitans (L.) Moench var. jaliscensis (Geenm.) H.S. Irwin \& Barneby - 1766, 4663, 6614, 9660

Chamaecrista nictitans (L.) Moench var. mensalis (Geenm.) H.S. Irwin \& Barneby - 5972

Chamaecrista nictitans (L.) Moench var. paraguariensis (Chod. \& Hassl.) H.S. Irwin \&

Barneby - 14660

Chamaecrista nictitans (L.) Moench var. pilosa (Benth.) H.S. Irwin \& Barneby - 10688, 11385

Chamaecrista rotundifolia (Pers.) Greene var. rotundifolia - 715, 1864, 6754 
Apéndice. Continuación.

Chamaecrista serpens (L.) Greene var. serpens - 1656

Chamaecrista serpens (L.) Greene var. wrightii (A. Gray) H.S. Irwin \& Barneby - 6862, 9542, 10536

Cynometra colimensis Britton \& Rose - 10335, 10893

Haematoxylum brasiletto Karst. - 3193, 3388, 5850, 9975, 10048

Parkinsonia aculeata L. - 3793, 5476, 5642

Parkinsonia hybrid - 6040

Senna alata (L.) Roxb. - 5660, 6904, 10887, 10956, 11764

Senna argentea (Kunth) H.S. Irwin \& Barneby - 6961

Senna atomaria (L.) H.S. Irwin \& Barneby - 5889

Senna centranthera H.S. Irwin \& Barneby - 15995

Senna foetidissima (G. Don) H.S. Irwin \& Barneby var. foetidissima - 1125, 6530 (tipo de

Cassia stenosepala L.B. Sm. \& B.G. Schub.) - 11876

Senna fruticosa Mill. - 1517, 4330, 7053, 10983, 11479, 11796, 12645

Senna holwayana (Rose) H.S. Irwin \& Barneby var. holwayana - 11297

Senna mollissima (Willd.) H.S. Irwin \& Barneby var. glabrata (Benth.) H.S. Irwin \&

Barneby - 10237, 10262, 10876, 11740, 11782, 12155

Senna multifoliolata (P.G. Wilson) H.S. Irwin \& Barneby var. eurystegia H.S. Irwin \&

Barneby - 14785

Senna multifoliolata (P.G. Wilson) H.S. Irwin \& Barneby var. metaxi H.S. Irwin \& Barneby 9945 (tipo), 10177

Senna multifoliolata (P.G. Wilson) H.S. Irwin \& Barneby var. multifoliolata - 12352 (tipo de Cassia multifoliolata P.G. Wilson)

Senna multiglandulosa (Jacq.) H.S. Irwin \& Barneby - 493, 2877, 4090, 6216, 13546

Senna nicaraguensis (Benth.) H.S. Irwin \& Barneby - 6758, 11425

Senna obtusifolia (L.) H.S. Irwin \& Barneby - 6021, 9360, 11439, 11617

Senna occidentalis Link - 4735, 5677, 9359, 11370, 11600

Senna pallida (Vahl) H.S. Irwin \& Barneby var. pallida - 11264, 11539, 12618, 12688, 16012

Senna pallida (Vahl) H.S. Irwin \& Barneby var. trichocraspedon (Sandwith) H.S. Irwin \&

Barneby - 2972 (tipo), 5582 (tipo), 5742 (tipo), 6144 (tipo), 8917, 9034

Senna pallida (Vahl) H.S. Irwin \& Barneby var. triquetripes H.S. Irwin \& Barneby - 11512

Senna pilifera (Vogel) H.S. Irwin \& Barneby - 1791, 4841, 7100, 11559

Senna pilifera (Vogel) H.S. Irwin \& Barneby var. subglabra (S. Moore) H.S. Irwin \& Barneby - 4816, 11746

Senna quinquangulata (L.C. Rich.) H.S. Irwin \& Barneby - 12547

Senna racemosa (Mill.) H.S. Irwin \& Barneby var. coalcomanica H.S. Irwin \& Barneby 12640,16105

Senna racemosa (Mill.) H.S. Irwin \& Barneby var. liebmannii (Benth.) H.S. Irwin \& Barneby - 3154

Senna racemosa (Mill.) H.S. Irwin \& Barneby var. sororia H.S. Irwin \& Barneby - 5894

Senna septemtrionalis (Viv.) H.S. Irwin \& Barneby - 662

Senna skinneri (Benth.) H.S. Irwin \& Barneby - 1447, 6114, 6622, 9177, 12112, 13008 
Apéndice. Continuación.

Senna uniflora (Mill.) H.S. Irwin \& Barneby - 1449, 5375, 5620, 9360, 11422, 11750

Senna wislizeni (A. Gray) H.S. Irwin \& Barneby var. pringlei (Rose) H.S. Irwin \& Barneby 6305,6612

Senna x floribunda (Cav.) H.S. Irwin \& Barneby - 8278

\section{Subfamilia Mimosoideae}

Acacia acatlensis Benth. - 4142, 5417, 5874, 7749, 7755

Acacia aff. acatlensis Benth. - 5955

Acacia amentacea DC. - 5698, 7274

Acacia cochliacantha Humb. \& Bonpl. ex Willd. - 1173, 2124, 5967, 5988, 6284, 6286,

6979, 7418, 7764, 7814, 7868, 9140, 10021, 10265, 10388

Acacia collinsii Saff. - 10333

Acacia coulteri Benth. - 3747

Acacia farnesiana (L.) Willd. - 1416, 3369, 5615, 13100

Acacia polyphylla DC. - 10785, 12548, 16110

Acacia macilenta Rose - 3355, 5507, 5701, 7269, 11745

Acacia pennatula (Schltdl. \& Cham.) Benth. - 601, 3426, 5934, 6022, 6973, 9986, 10040

Acacia picachensis T.S. Brandegee - 6279, 6872, 8141

Acacia riparia Kunth var. acapulcensis L. Rico, ined. - 5748

Acaciella angustissima (Mill.) Britton \& Rose var. angustissima - 197, 5088, 7162, 7778,

8621, 8780, 9489, 10896

Acaciella angustissima (Mill.) Britton \& Rose var. filicioides (Cav.) L. Rico - 892, 5734,

7963, 12415, 17233

Acaciella hartwegii (Benth.) Britton \& Rose - 1291, 1423, 4744, 8183, 9418

Acaciella painteri Britton \& Rose var. houghii (Britton \& Rose) L. Rico - 5367, 5377, 8142, 8763A, 8856, 11487

Acaciella painteri Britton \& Rose var. painteri - 8184, 8763B, 12453

Acaciella igualensis Britton \& Rose - 2500, 5412, 6263, 8016, 8021, 8027, 8136, 10538, 11248

Acaciella sousae (L. Rico) L. Rico - 9541 (tipo)

Acaciella tequilana (S. Watson) Britton \& Rose - 1945, 4852, 5405

Acaciella villosa (Sw.) Britton \& Rose - 10815

Adenopodia gymnantha Brenan - 12588 (tipo)

Albizia tomentosa (Micheli) Standl. - 3815, 3966, 6046, 9980, 10326

Albizia sp. 7493

Calliandra bijuga Rose - 7806, 12726

Calliandra caeciliae Harms - 9154

Calliandra hintonii Barneby - 4099

Calliandra hirsuta (G. Don) Benth. - 12711

Calliandra houstoniana (Miller) Standl. - 6772, 6967, 10685

Calliandra houstoniana (Miller) Standl. var. acapulcensis (Britton \& Rose) Barneby - 11044 
Apéndice. Continuación.

Calliandra houstoniana (Miller) Standl. var. anomala (Kunth) Barneby - 903, 1545, 10503, 11877, 12712

Calliandra houstoniana (Miller) Standl. var. calothyrsus (Meissner) Barneby - 3236

Calliandra houstoniana (Miller) Standl. var. houstoniana - 11457, 12476

Calliandra tergemina (L.) Benth. var. tergemina - 10183

Chloroleucon mangense (Jacq.) Britton \& Rose - 11647

Desmanthus bicornutus S. Watson - 4795, 6885

Desmanthus interior (Britton \& Rose) Bullock - 6855, 8218

Desmanthus virgatus (L.) Willd. - 5440

Entada polystachya (L.) DC. var. polystachya - 925, 1478, 4274, 4781, 5945, 9148, 11379,

Enterolobium cyclocarpum (Jacq.) Griseb. - 3416, 3641

Havardia acatlensis (Benth.) Britton \& Rose - 725, 928, 4140, 5658, 9989

Inga hintonii Sandwith - 7617 (tipo)

Inga micheliana Harms - 456, 4182, 6171, 7667, 8103, 8232, 8978, 11202

Inga oerstediana Benth. - 10746

Inga vera Willd. subsp. eriocarpa (Benth.) J. Leon - 373, 3159, 3981, 5527, 5572, 5911, 6184, 6205, 6232, 6483, 7349, 8977, 9089, 9248, 9259, 9997, 10074

Leucaena leucocephala (Lam.) de Wit subsp. glabrata (Rose) S. Zárate - 3119, 5138, 5408, 7287

Leucaena esculenta Benth. - 1965, 2349, 5745, 7417, 13371

Leucaena macrophylla Benth. subsp. macrophylla - 2256, 5098, 7493, 8611, 10985, 12733, 16182

Leucaena trichandra (Zucc.) Urb. - 3970, 7993

Leucaena aff. trichandra (Zucc.) Urb. - 6843

Lysiloma acapulcense (Kunth) Benth. - 464, 705, 2784, 3760, 3838, 5904, 10006, 10025, 14250

Lysiloma auritum (Schltdl.) Benth. - 10132

Lysiloma divaricatum (Jacq.) Macbride - 14586

Lysiloma microphyllum Benth. - 763, 933, 4269, 6125, 9162

Lysiloma tergeminum Benth. - 929, 4180, 5968, 6607, 10383, 11980

Mimosa acantholoba Humb. \& Bonpl. ex Willd. var. eurycarpa (B.L. Rob.) Barneby - 14541

Mimosa aculeaticarpa Ortega - 2853, 6814, 7446, 7693, 17106

Mimosa adenantheroides (M. Martens \& Galeotti) Benth.- 1088, 1323, 7835, 12709, 15023, 15090

Mimosa affinis B.L. Rob. - 1600, 1756, 4743, 5995

Mimosa albida Humb. \& Bonpl. ex Willd. - 1415, 1642, 6540, 9424, 10639, 10640, 11485, 11971

Mimosa albida Humb. \& Bonpl. ex Willd var. albida - 4671

Mimosa albida Humb. \& Bonpl. ex Willd var. glabrior B.L. Rob. - 10904

Mimosa caerulea Rose - 1702, 6546, 7017, 8217, 8231, 8756, 8894, 17408

Mimosa camporum Benth. - 6628

Mimosa diplotricha C. Wright ex Sauvalle var. diplotricha - 2290, 9666 
Apéndice. Continuación.

Mimosa diplotricha C. Wright ex Sauvalle var. odibilis Barneby - 6036

Mimosa egregia Sandwith - 6296, 6314 (tipo), 6907 (tipo), 7887, 8035, 8519

Mimosa galeottii Benth. - 898, 6248, 6256, 7185, 7197, 8427

Mimosa guatemalensis (Hook. \& Arn.) Benth. - 2481, 5102, 5864, 13372

Mimosa lacerata Rose - 3737, 5791, 6865

Mimosa nanchititlana R. Grether \& Barneby - 4381, 6551, 7337, 8068, 8889

Mimosa occidentalis Britton \& Rose - 8485

Mimosa pigra L. var. pigra -10232

Mimosa polyantha Benth. - 778, 3957, 4299, 5793, 5884, 6131, 6298, 6940, 7862, 7874, 8516, 8525, 11979

Mimosa quadrivalvis L. var. diffusa (Rose) Beard ex Barneby - 12235

Mimosa rhododactyla B.L. Rob. var. benthamii (Macbride) Barneby - 1237, 1644, 5410, $6405,6831,8224,8890,9427,12126,13148$

Mimosa robusta R. Grether - 1300, 1475, 10628, 10836, 11001

Mimosa somnians Humb. \& Bonpl. ex Willd. var. somnians - 11337

Mimosa tricephala Cham. \& Schltdl. var. xanti (A. Gray) T. Chehaibar \& R. Grether - 3802, 5737, 5885, 5984, 5987, 6864, 6915, 7055, 7838, 9058, 9156, 9161, 10510

Mimosa sp. - 5839

Neptunia plena (L.) Benth. - 6495

Neptunia prostrata (Lam.) Baill. - 6652

Piptadenia flava (Spreng. ex DC.) Brenan - 4506, 5468, 6635, 6705, 9157

Piptadenia obliqua (Pers.) Macbr. subsp. obliqua - 6107, 9063, 9991,10321, 16102

Pithecellobium dulce (Roxb.) Benth. - 453, 3002, 3192, 3931, 5430, 5524, 5966, 7591

Pithecellobium dulce (Roxb.) Benth. vel aff. - 5812

Pithecellobium lanceolatum (Humb. \& Bonpl. ex Willd.) Benth. - 5614, 5819

Pithecellobium oblongum Benth. - 8515

Pithecellobium seleri Harms - 5865, 7756, 10871

Prosopis juliflora (Sw.) DC. - 10886

Prosopis laevigata (Humb. \& Bonpl. ex Willd.) M.C. Johnst. - 3803, 5471, 7813

Zapoteca formosa (Kunth) H. Hern. subsp. formosa - 6466, 6878, 7094, 11626

Zapoteca formosa (Kunth) H. Hern. subsp. rosei (Wiggins) H. Hern. - 4433, 6545, 9191, 13053

Zapoteca portoricensis (Jacq.) H. Hern. subsp. portoricensis - 2019

Zapoteca tetragona (Willd.) H. Hern. - 2207, 7101, 10847, 11059, 11577, 11780, 12274, 12331

Zygia conzattii (Standl.) Britton \& Rose - 10782

Subfamilia Papilionoideae

Aeschynomene americana L. var. americana -9668

Aeschynomene americana L. var. flabellata Rudd - 1619, 1984, 2057, 2234, 5992, 6654, 9503, 13287 
Apéndice. Continuación.

Aeschynomene americana L. var. glandulosa (Poir.) Rudd - 1604, 10922

Aeschynomene compacta Rose - 6936, 7872

Aeschynomene hintonii Sandwith - 6104 t(ipo), 10644

Aeschynomene histrix Poir. var. densiflora (Benth.) Rudd - 4582

Aeschynomene palmeri Rose - 14604, 16060, 16163

Aeschynomene paniculada Willd. ex Vog. - 5044, 11396

Aeschynomene paucifoliolata Micheli - 1764, 1997, 4784, 6708, 6938, 7059

Aeschynomene petraea B.L. Rob. var. madrensis (Micheli) Rudd - 10395, 11332

Aeschynomene petraea B.L. Rob. var. petraea - 457

Aeschynomene rudis Benth. -16106

Aeschynomene scabra G. Don - 1930, 4957, 6679, 9667, 11662

Aeschynomene unijuga (M E. Jones) Rudd - 2410, 11670

Aeschynomene villosa Poir. var. longifolia (Micheli) Rudd - 9669, 10747

Aeschynomene villosa Poir. var. villosa - 8608

Amicia zygomeris DC. - 808, 1816, 2047, 2554, 5087, 7012, 9573, 11482, 12267, 13352

Andira inermis (W. Wright) DC. subsp. inermis - 9978, 14091

Apoplanesia paniculata Presl - 10856, 12116, 12606, 16034

Astragalus ervoides Hook. \& Arn. - 12727

Astragalus hintonii Barneby - 539, 13240, 13414

Astragalus painteri Rose - 5067, 5232, 8912

Astragalus purpusii M.E. Jones - 17202

Astragalus tolucanus B.L. Rob. \& Seaton - 1900

Ateleia arsenii Standl. - 407, 3421, 3526, 4240, 5286, 6093, 6158, 7621

Brongniartia benthamiana Hemsl. - 10435

Brongniartia cf. lupinoides Standl. - 4067

Brongniartia cuneata L.B. Sm. \& B.G. Schub. - 6311 (tipo)

Brongniartia funiculata L.B. Sm. \& B.G. Schub. - 5397 (tipo), 8019, 8727

Brongniartia glabrata Hook. \& Arn. - 11619, 14025

Brongniartia gracilis Hemsl. - 6230

Brongniartia inconstans S. Watson - 10035

Brongniartia intermedia Moric. - 890

Brongniartia lupinoides Standl. - 11973

Brongniartia podalyrioides Kunth subsp. podalyrioides - 3211, 5387, 5854, 9831

Brongniartia proteranthera L.B. Sm. \& B.G. Schub.- 5947 (tipo)

Calopogonium caeruleum (Benth.) Sauv. - 3134, 5584, 5829, 11645

Calopogonium galactioides Benth. ex Hemsl. - 5404, 5579

Calopogonium mucunoides Desv. - 2247, 5156, 10905

Canavalia hirsutissima J.D. Sauer - 11053 (tipo)

Canavalia mexicana Piper - 5341, 6910, 11618, 12237

Canavalia villosa Benth. - 2082, 2288, 5335, 8875, 9911, 10826, 11673, 11860, 14037 , 16263,17433

Centrosema pubescens Benth. - 9623 
Apéndice. Continuación.

Centrosema sagittatum (Humb. \& Bonpl. ex Willd.) Brandegee - 5006, 10825, 10914, 11560

Centrosema virginianum (L.) Benth. - 4792, 4994, 7060, 9623A

Cleobulia crassistyla R. H. Maxwell - 9900 (tipo), 10791 (tipo), 14996 (tipo).

Clitoria mexicana Link - 1166, 4968, 9892, 10751

Clitoria polystachya Benth. - 1371, 4523, 5334, 8125

Clitoria aff. polystachya Benth. - 6377, 8001, 8597, 10478

Clitoria sp. - 1528, 4447

Cologania broussonetii (Balb.) DC. - 751, 1580, 6069, 9587, 10656, 17415

Cologania cf. biloba Nichols. - 2242

Cologania cf. pulchella Kunth - 11483, 11837, 12204

Cologania deamii Fernald - 10394

Cologania hirta Rose - 2767, 11262, 11694, 14947

Cologania pulchella Kunth - 1023A, 13115

Coursetia caribaea (Jacq.) Lavin var. caribaea - 5191, 6914, 8490, 8497, 8740, 11463

Coursetia glandulosa A. Gray - 3350, 5528, 5663, 5836

Coursetia hintonii Rudd - 2983, 3404, 7051 (tipo)

Coursetia sp. - 6135

Crotalaria acapulcensis Hook. \& Arn. - 1626, 4854, 6757, 9563, 12638, 13126

Crotalaria bupleurifolia Cham. \& Schltdl. var. bupleurifolia - 1730, 5424

Crotalaria bupleurifolia Cham. \& Schltdl. var. robusta (H. Senn) Windler - 2686 (tipo), 4595, 5580, 11708

Crotalaria cajanifolia Kunth - 670, 1870, 10352, 10617, 10843, 10912, 11581, 12238

Crotalaria eriocarpa Benth. - 1290, 10754

Crotalaria filifolia Rose - 1474, 4717, 4835, 4964, 6498, 8176, 9464

Crotalaria incana L. var. australis Griseb. - 4798, 6744

Crotalaria longirostrata Hook. \& Arn. - 1421, 8306, 10920, 11357, 12725, 13055, 16157

Crotalaria micans Link - 12252, 12723

Crotalaria mollicula Kunth - 9261

Crotalaria pumila Ortega - 1636, 2059, 2652, 4658, 5540, 6041, 8291, 10902, 11395 , 11580, 12198, 13145

Crotalaria quercetorum Brandegee - 11413

Crotalaria rotundifolia Windler var. vulgaris Windler - 2454

Crotalaria tuerckheimii H. Senn var. macrantha H. Senn - 5068 (tipo)

Dalbergia calycina Benth. - 591, 881, 3971, 6194, 7931

Dalbergia glomerata Hemsl. - 815, 3177, 5577, 7248

Dalbergia melanocardium Pittier - 761, 3935, 4322

Dalea carthagenensis (Jacq.) Macbride var. capitulata (Rydb.) Barneby- 7564, 8572

Dalea cliffortiana Willd. - 1630, 2001, 2217, 2615, 2636, 4755, 5184, 5292, 6968, 9391, 9602, 9909, 11501, 12234, 13269

Dalea cuniculo-caudata P. G. Wilson - 1590, 2168, 2333 (tipo), 13462

Dalea elata Hook. \& Arn. 3338, 5364 
Apéndice. Continuación.

Dalea foliolosa (Aiton) Barneby var. citrina (Rydb.) Barneby - 1943, 2257, 6683, 7580, 9530, 9586, 12322

Dalea hintonii Sandwith - 5314 (tipo), 8872

Dalea leporina (Aiton) Bullock - 1686, 1888, 2273, 5048, 7786, 9665, 11992

Dalea leucostachys A. Gray - 11549

Dalea leucostachys A. Gray var. eysenhardtioides (Hemsl.) Barneby- 2007, 2086, 12401, 12777

Dalea lutea (Cav.) Willd. var. gigantea (Rydb.) Barneby - 975, 1846

Dalea lutea (Cav.) Willd. var. lutea - 17183

Dalea obovatifolia Ortega var. uncifera (Schltdl. \& Cham.) Barneby - 5317

Dalea obreniformis (Rydb.) Barneby - 4982, 8352, 15561

Dalea plantaginoides Barneby - 15665

Dalea polystachya (Sessé \& Moc.) Barneby - 5340, 12780

Dalea sericea Lag. - 1944, 13425, 17453

Dalea tomentosa Willd. var. psoraloides (Moric.) Barneby - 2232, 9598, 11527, 12327

Dalea urceolata Greene var. tripetala (P.G. Wilson) Barneby - 13351 (tipo de

Petalostemon tripetalus P.G. Wilson)

Dalea sp. - 2327, 2720, 11840, 13397, 14713, 14822, 15152, 15420

Desmodium affine Schltdl. - 6717

Desmodium alamanii DC. - 8297

Desmodium amplifolium Hemsl. -11092, 11282, 11696

Desmodium angustifolium (Kunth) DC. var. angustifolium - 1611, 2235, 2301, 2505, 4492, 4827, 9610, 11498

Desmodium barbatum (L.) Benth. \& Oerst. - 4952

Desmodium bellum (S.F. Blake) B.G. Schub. - 2920

Desmodium cf. infractum DC. - 1496, 4749, 6626

Desmodium cinereum (Kunth) DC. - 9657, 10918, 13249

Desmodium cordistipulum Hemsl. - 2818, 15472

Desmodium densiflorum Hemsl. - 271, 2834, 8939, 13533

Desmodium ghiesbreghtii Hemsl. - 1734

Desmodium hartwegianum Hemsl. var. amans (S. Watson) B.G. Schub. - 1621

Desmodium distortum (Aubl.) J.F. Macbr. - 8363, 8573, 14864

Desmodium hookerianum D. Dietr. - 10940, 11686

Desmodium intortum (Mill.) Urb. var. apiculatum B.G. Schub. - 271, 2834, 8939, 10944, 11524,16130

Desmodium jaliscanum S. Watson - 1862, 2020, 2528, 6771, 13395

Desmodium macrostachyum Hemsl. - 12159

Desmodium michelianum (Schindl.) B.G. Schub. \& McVaugh - 2706

Desmodium molliculum (Kunth) DC. - 15473

Desmodium neomexicanum A. Gray - 1927, 2174

Desmodium nicaraguense Oerst. - 2523, 5337, 8568 
Apéndice. Continuación.

Desmodium orbiculare Schltdl. var. rubricaule (Rose \& Painter) B.G. Schub. \& McVaugh - 1055, 9258, 11348

Desmodium plicatum Schltdl. \& Cham. - 3000, 10828, 11638

Desmodium prehensile Schltdl. - 9661, 11031, 11511

Desmodium pringlei S. Watson - 1622, 1894

Desmodium procumbens (Mill.) Hitchc. var. exiguum (A. Gray) B.G. Schub. - 6693, 6695

Desmodium procumbens (Mill.) Hitchc. var. longipes (Schindl.) B.G. Schub. - 5187

Desmodium procumbens (Mill.) Hitchc. var. procumbens - 1928, 1950, 6681, 8494, 8517, $8729,9534,10863$

Desmodium procumbens (Mill.) Hitchc. var. transversum (B.L. Rob. \& Greenm.) B.G.

Schub. - 1754

Desmodium retinens Schltdl. - 13303

Desmodium scorpiurus (Sw.) Desv. - 3945, 5629, 6037, 11419

Desmodium sericophyllum Schltdl. -11458, 12177

Desmodium skinneri Benth. ex Hemsl. - 192, 2942, 3236, 5290

Desmodium strobilaceum Schltdl. - 9390, 9568, 13286

Desmodium sumichrastii (Schindl.) Standl. - 2822, 5338, 8690, 8734, 8871, 10681, 11144, 12771

Desmodium sylvicola Brandegee - 2866

Desmodium tortuosum (Sw.) DC. - 5480, 6892, 8523, 8728, 11448

Desmodium sp. - 361, 2279, 8810, 9447, 10724, 11141, 11351, 11553, 11667, 11696,

$13395,15245,15474,15475,15680,15781,17411,17430,17454$

Dioclea sp. nov. ined. - 11737

Diphysa americana (Mill.) M. Sousa - 5221, 7322, 11755

Diphysa minutifolia Rose - 5796, 10031

Diphysa puberulenta Rydb. - 16205

Diphysa racemosa Rose - 194, 312, 2975, 3011, 8877, 13564

Diphysa robinioides Benth. - 11269

Diphysa sennoides Benth. ex Benth. \& Oerst. - 1015

Diphysa suberosa S. Watson - 718, 770, 10451

Eriosema diffusum (Kunth) DC. - 1592

Eriosema grandiflorum (Schltdl. \& Cham.) G. Don - 2495, 5106, 5293, 9797, 11494, 12470

Eriosema longicalyx Grear - 8935

Eriosema multiflorum B.L. Rob. - 1988

Eriosema palmeri S. Watson var. palmeri - 13124

Eriosema pulchellum (Kunth) DC. - 1181, 10462, 10844

Erythrina breviflora DC. - 527, 1861, 1924, 11513, 12314, 12468, 13382

Erythrina lanata Rose var. lanata - 3397, 6095, 7732, 10030, 12084

Erythrina leptorhiza DC. - 15402, 17163

Erythrina mexicana Krukoff - 5333 (tipo), 6157, 14708

Eysenhardtia polystachya Sarg. - 380, 1143, 1223, 8015, 9247, 12738, 13060 
Apéndice. Continuación.

Galactia incana Standl. - 1286, 1652, 10476, 11378

Galactia multiflora B.L. Rob. - 1180, 6559

Galactia striata Urb. - 4829, 5388, 11409

Galactia viridiflora Standl. - 6893, 13375

Gliricidia sepium (Jacq.) Steud. - 3139, 3331, 5713, 5869, 10230, 11246, 12581, 12735, 16234, 16288

Harpalyce formosa DC. - 4083, 6146, 6233

Harpalyce sousai M.T.K. Arroyo - 3444, 4114, 4209, 5411, 5902, 6995

Hybosema ehrenbergii Harms - 5928, 7983

Indigofera cuernavacana Rose - 8090

Indigofera densiflora M. Martens \& Galeotti - 1583, 8239, 17404

Indigofera hartwegii Rydb. - 1052, 1236, 6355, 6364, 6999, 9135, 10477, 11885

Indigofera jaliscensis Rose - 6767, 11455

Indigofera langlassei Rydb. - 11606

Indigofera mucronata Willd. ex Spreng. - 6421, 8116, 10906, 12079

Indigofera palmeri $\mathrm{S}$. Watson - 1217, 5856, 6112, 7829, 7850, 7884

Indigofera suffruticosa Mill. - 1178, 5452, 10193, 11055, 12236

Indigofera thibaudiana DC. - 1698, 2018, 7441, 12664, 12728

Indigofera sp. - 11045

Lonchocarpus balsensis M. Sousa \& J.C. Soto - 7753

Lonchocarpus caudatus Pittier - 1473, 8179

Lonchocarpus constrictus - 5861, 5986, 6300, 10349, 10387

Lonchocarpus guatemalensis Benth. - 11729

Lonchocarpus hintonii Sandwith - 5936 (tipo), 6508 (tipo)

Lonchocarpus lanceolatus Benth. - 6649, 7281, 7288, 8495, 10540, 12546, 16272

Lonchocarpus longipedicellatus Pittier - 10379, 10384, 10459

Lonchocarpus longipedunculatus M. Sousa \& J.C. Soto - 5876

Lonchocarpus rugosus Benth. - 5891, 6325

Lonchocarpus schubertiae M. Sousa - 3742, 5523, 5937, 5942, 5946, 6290, 6959, 7041, 10386

Lonchocarpus venustus P.J. Hermann - 7763

Lonchocarpus sp. - 3774

Lotus angustifolius Gouan - 3422, 3422A, 4877, 5226, 8773

Lotus repens Moc. \& Sessé ex G. Don - 805, 6255

Lupinus aschenbornii S. Shauer var. cervantesii C.P. Sm. - 4626, 8290 (tipo), 8340 (tipo)

Lupinus barkeri Lindl. - 2537, 5407, 6151, 6760, 7063, 7080, 8260, 8280, 8281, 8304, 8305, 8307, 8308, 8350, 8380, 11414, 14942

Lupinus cacuminis Standl. - 17002, 17038

Lupinus decaschistus C.P. Sm. - 14786 (tipo)

Lupinus diehlii M.E. Jones - 13565, 15124

Lupinus ehrenbergii Schltdl. - 8261, 8268, 8269, 8270, 8289, 8302, 8303, 8316, 8318, $8321,8960,8965,8994,9023,13450,15403$ 
Apéndice. Continuación.

Lupinus ehrenbergii Schltdl. var. barberi C.P. Sm. - 13362, 13493

Lupinus elegans Kunth - 8925, 8947, 9648, 9709, 9754, 9858, 9943, 11814, 12750, 12752

Lupinus elegans Kunth var. campestris (Schltdl. \& Cham.) C.P. Sm. - 11939

Lupinus elegans Kunth var. exaltatus (Zucc.) C.P. Sm. - 5924, 7475, 8288, 8771, 8809, 8940, 8943, 8944, 8991, 9006, 9929, 10158, 11087, 12392, 12671 (tipo), 12748, 12753, $12786,12795,13179,13347,13466,13509,14093,14771$

Lupinus elegans Kunth var. pulchellus (Sweet) C.P. Sm. - 8677, 12775

Lupinus elegans Kunth var. quercetorum C.P. Sm. - 13535 (tipo)

Lupinus fictomagnus C.P. Sm. - 12633 (tipo)

Lupinus hintonii C.P. Sm. - 781 (tipo), 8336, 9019, 11932

Lupinus montanus Kunth - 8244, 8317, 9031

Lupinus monteportae C.P. Sm. - 12720 (tipo)

Lupinus octablomus C.P. Sm. - 14521 (tipo)

Lupinus persistens Rose praeclarus C.P. Sm. - 8243, 8315 (tipo), 13237

Lupinus potosinus Rose - 321

Lupinus quercuum C.P. Sm. - 8362 (tipo)

Lupinus quercuum C.P. Sm. var. perflexolobius C.P. Sm. - 13285 (tipo)

Lupinus rhodanthus C.P. Sm. - 13117 (tipo)

Lupinus simulans Rose var. abietum C.P. Sm. - 8937 (tipo), 10731, 13414, 13505

Lupinus splendens Rose - 8360

Lupinus squamaecaulis C.P. Sm. - 9937 (tipo), 10154, 12345, 12346, 12755

Lupinus taurimortuus C.P. Sm. - 11088 (tipo)

Machaerium isadelphum (E. Mey.) Amshoff - 3140, 3645

Machaerium salvadorense (Donn. Sm.) Rudd - 11726, 16283

Macroptilium atropurpureum (Sessé \& Moc. ex DC.) Urb. - 2269, 3583, 4745, 5107, 5415, $5575,6045,6671,6734,6888,8505,11775,13207$

Macroptilium gibbosifolium (Ortega) A. Delgado - 1605, 12221

Macroptilium longepedunculatum (Mart. ex Benth.) Urb. - 5007, 6866

Macroptilium pedatum (Rose) Maréchal \& Baudet - 6602, 8512

Marina diffusa (Moric.) Barneby - 2267, 11255

Marina gracilis Liebm. - 1462, 1750, 4776A, 6665, 9409, 13294

Marina neglecta (B. L. Rob.) Barneby var. elongata (Rose) Barneby - 5846 (tipo)

Marina nutans (Cav.) Barneby - 124, 2281, 2354, 2541, 2692, 8658, 10997, 11586, 13376, 13428, 17471

Marina spiciformis (Rose) Barneby - 1755, 1938, 13273

Marina unifoliata (B. L. Rob. \& Greenm.) - 6035, 9719, 16158

Mucuna sloanei Fawc. \& Rendle - 8759, 10804, 10934

Myrospermum frutescens Jacq. - 7291

Myroxylon balsamum (L.) Harms - 12296, 12298, 12540

Nissolia fruticosa Jacq.- 14546

Nissolia hintonii Sandwith - 2334 (tipo)

Nissolia hirsuta DC. - 1197, 4319, 6280, 6692, 9142 
Apéndice. Continuación.

Nissolia leiogyne Sandwith - 6291 (tipo)

Nissolia nelsoni Rose - 6709, 8163, 12682

Pachyrhizus erosus (L.) Urb. - 1205, 4424, 9367, 9540, 11486, 11776

Phaseolus leptostachyus Schltdl. - 2266, 2287, 6642, 9382, 9485, 9567, 10601, 10827, 10947, 13163

Phaseolus coccineus L. - 623, 2006, 2310, 9755, 12261, 17425

Phaseolus coccineus L. subsp. formosus Kunth - 8279, 13276

Phaseolus pauciflorus Sessé \& Mociño ex G. Don - 1555, 1588, 4653, 4708

Phaseolus hintonii A. Delgado - 6728 (tipo)

Phaseolus leptostachyus Schltdl. - 2266, 2287, 6642, 9382, 9485, 9567, 10601, 10827, 10947, 13163

Phaseolus lunatus L. - 3100, 3121, 5383, 5439, 11742, 11771

Phaseolus marechalii A. Delgado - 17462

Phaseolus microcarpus M. Martens - 8584

Phaseolus perplexus A. Delgado - 4714, 4799, 12191

Phaseolus pedicellatus Benth. - 542, 2375, 11142, 17245

Phaseolus xolocotzii A. Delgado - 2484

Phaseolus tenellus Piper - 4719, 6528

Phaseolus vulgaris L. - 5605, 11551, 11567, 11684

Phaseolus sp. - 4796, 5605, 8245

Piscidia carthagenensis Jacq. - 3578, 5626, 5803, 7524, 9995, 10008, 11802

Piscidia grandifolia (Donn. Sm.) I.M. Johnst. var. glabresces Sandwith - 1193 (tipo), 5419 (tipo), 8079, 8603, 10518, 13057

Platymiscium jejunum B.B. Klitgaard - 14001 (tipo)

Platymiscium lasiocarpum Sandwith - 3174 (tipo), 3389, 5396, 7320, 7406, 7503, 7507, 7532, 552, 7922

Poiretia scandens Vent. - 5142, 5361, 6751

Pterocarpus orbiculatus DC. - 3607, 3965, 5699, 5881, 9985, 9987, 11801

Ramirezella micrantha A. Delgado \& Ochoterena-Booth - 14658

Ramirezella strobilophora (B.L. Rob.) Rose var. pubescens (Rose) Maréchal, Mascherpa \& Stainer - 3441, 5574, 7415, 7782, 7897, 7935, 11634, 16138

Ramirezella sp. - 1989, 6989, 9350, 10927

Rhynchosia discolor M. Martens \& Galeotti - 3058, 3197, 7342, 8659, 15778

Rhynchosia edulis Griseb. - 1182, 2258, 7336, 10569

Rhynchosia minima DC. - 2230, 3163, 3786, 5434, 10945

Rhynchosia precatoria (Willd.) Kunth -5399, 14036

Rhynchosia sp. - 2280, 3074

Sesbania macrocarpa Muhl. ex Raf. - 1985, 4729, 5618

Sphinctospermum constrictum (S. Watson) Rose - 4732, 6474, 6507

Stylosanthes guianensis (Aubl.) Sw. - 11456

Stylosanthes humilis Kunth - 6985

Stylosanthes subsericea S.F. Blake - 1931, 4767 
Apéndice. Continuación.

Swartzia simplex (Sw.) Spreng. var. ochnacea (DC.) R.S. Cowan - 10222, 10632, 15951, 16230

Tephrosia conzattii Standl. - 1297, 5273, 6998

Tephrosia cuernavacana J.F. Macbr. - 10485

Tephrosia diversifolia J.F. Macbr. - 12719

Tephrosia macrantha B.L. Rob. \& Greenm. ex Pringle - 5099, 5172, 11558

Tephrosia mexicana C.E. Wood - 2698 (tipo)

Tephrosia multifolia Rose - 11006, 16180

Tephrosia nicaraguensis Oerst. - 1278

Tephrosia nitens Benth. - 11037

Tephrosia piscatoria Pers. - 6645

Tephrosia pogonocalyx C.E. Wood - 3101 (tipo)

Tephrosia purpurea Pers. - 5180

Tephrosia rhodantha Brandegee - 1921, 6603, 10845, 11403, 11641

Tephrosia singapou (Buchoz) A. Chev. - 4683, 8114

Tephrosia tenella A. Gray - 16117

Tephrosia vernicosa C.E. Wood - 9950 (tipo)

Tephrosia vicioides Schltdl. - 1939, 5096

Teramnus uncinatus Sw. - 3830

Trifolium amabile Kunth - 1311, 1374, 2091

Trifolium hemsleyi Lojac. - 4630, 4905

Trifolium mexicanum Hemsl. - 12419, 13108

Trifolium wormskioldii Lehm. var. ortegae (Greene) Barneby - 796, 1390, 13114

Vicia cracca L. - 2812, 8284, 13422, 13452

Vicia hirsuta (L.) A. Gray - 4895

Vicia humilis Kunth - 4912, 6840, 8897, 17005

Vicia pulchella Kunth - 17041

Vicia sativa L. - 396, 2456

Vigna adenantha (G. Mey.) Maréchal, Mascherpa \& Stainer- 1929, 10960, 11491, 11568, 11709

Vigna luteola Benth. - 5541, 5646, 5654, 6002

Vigna speciosa (Kunth) Verdc. - 11628, 11738

Vigna linearis (Kunth) Maréchal, Mascherpa \& Stainer - 4516, 4814

Zornia diphylla (L.) Pers. - 1655, 1665, 4499, 4756, 4868, 9179, 10767, 11043, 11640, 12244

Zornia reticulata Sm. - 1803, 1883, 9571, 11427 